\title{
Géolinguistique
}

16 | 2016

Varia

\section{Nommer l'animal déviant. Le cas de la vache stérile en domaine occitan}

Name the Deviant Animal. The Case of the "Infertile Cow" in the Occitan Domain

\section{Albert Malfatto}

\section{(2) OpenEdition}

\section{Journals}

\section{Édition électronique}

URL : http://journals.openedition.org/geolinguistique/559

DOI : 10.4000/geolinguistique.559

ISSN : 2650-8176

\section{Éditeur}

UGA Éditions/Université Grenoble Alpes

\section{Édition imprimée}

Date de publication : 1 décembre 2016

Pagination : 207-224

ISBN : 978-2-84310-342-1

ISSN : 0761-9081

\section{Référence électronique}

Albert Malfatto, "Nommer l'animal déviant. Le cas de la vache stérile en domaine occitan »,

Géolinguistique [En ligne], 16 | 2016, mis en ligne le 15 février 2019, consulté le 29 octobre 2020. URL http://journals.openedition.org/geolinguistique/559; DOI : https://doi.org/10.4000/geolinguistique. 559 


\title{
Nommer l'animal déviant. Le cas de la vache stérile en domaine occitan
}

\author{
Albert Malfatto \\ Université Nice-Sophia-Antipolis, \\ UMR 7320 : laboratoire Bases, Corpus, Langage
}

\section{Résumé}

Le présent article se propose d'analyser un ensemble de données dialectales occitanes relatives au concept de vache stérile. Ce faisant, il rend compte des liens qui unissent l'homme et l'animal domestique considéré, de par sa déviance, comme inutile par son propriétaire. L'analyse d'un corpus dialectal offrant une vision de la variation lexicale en vigueur, des processus morphologiques à l'œuvre et des diverses filiations étymologiques attestées, met en lumière les visions du monde particulières, les ressorts cognitifs, les liens culturels et représentationnels qui lient l'animal infécond, sa dénomination et l'éleveur.

\section{Mots-clés}

Lexicologie, dialectologie, zoonymes, motivation sémantique, dialectes occitans, animaux domestiques.

\section{Abstract}

This paper aims to give an analysis of some lexical datas associated to the concept of "infertile cow" in Occitan dialects. By doing this, it shows the relations between a useless or deviant domesticated mammal and it's owner. Taking into consideration a dialectal corpus of lexical datas, analysing lexical variation, morphological process and etymological connections, it points out wolrdviews, cultural and cognitives links that bond the animal, its name and its breeder. 


\title{
Keywords
}

Lexicology, Dialectology, zoonyms, semantical motivation, Occitan dialects, domesticated animals.

\author{
«On écarte les animaux épuisés et vieillis, \\ devenus impropres à la propagation, et surtout \\ les jeunes vaches stériles, qui tiennent la place \\ de bêtes fécondes [...].» \\ Columelle, De l'économie rurale, \\ Liv. VI, XXII.
}

\section{Introduction}

Au sein du bétail, une créature contrevenant au bon fonctionnement du troupeau - que ce soit par son manque de productivité, son état ou une quelconque tare jugée impropre - sera, en plus d'être dépréciée, méprisée voire supprimée, distinguée de ses congénères par une appellation propre, visant à marquer l'individu. Car il n'est pas rare, malgré une sélection des gènes active depuis l'émergence de l'élevage, résultat d'une sélection artificielle des caractéristiques et des comportements animaliers, que certains individus disposent dès la naissance - ou acquièrent durant leur existence - des particularités physiques ou comportementales considérées comme néfastes par les éleveurs et faisant d'eux des animaux de peu de valeur. Parmi ceux-ci, nous avons choisi d'étudier le cas complexe des bovins femelles, et, plus particulièrement, de celles touchées par cette tare bien spécifique qui les voue aux gémonies : la stérilité.

Nous tenterons de voir si le rapport privilégié qui unit l'homme et l'animal domestique change lorsque ce dernier devient déviant, acquiert une tare, ne correspond pas ou plus aux standards ou aux espérances de son propriétaire. La prise en compte de la motivation sémantique et de la variation diatopique et diachronique permettront de mettre en lumière certaines visions du monde et représentations cognitives en vigueur chez les locuteurs de communautés occitanophones.

\section{Corpus sélectionné et concept étudié}

Bien que l'étude proposée ici ne se veuille pas exhaustive - nous verrons certaines des raisons qui expliquent pourquoi une telle entreprise nécessiterait un espace plus important - elle se base sur une sélection de 286 données issues des divers atlas linguistiques et ethnographiques de la France 
par région et regroupées au sein du Thesaurus Occitan ${ }^{1}$ (THESOC), base de données dialectale regroupant l'ensemble des données occitanes publiées dans les atlas linguistiques et ethnographiques de la France par région, ainsi que diverses données supplémentaires recueillies au fil des ans par les membres de l'équipe de dialectologie du laboratoire Bases Corpus Langage de l'Université de Nice.

Comme de coutume, l'analyse des données issues de telles sources nécessite la pleine considération des écueils qu'elles induisent. Il a été évoqué par ailleurs, et nous reprenons ici les intitulés en les espérant transparents, les trois principaux que sont «l'absence de certaines catégories notionnelles», «l'utilisation de périphrases et de questions diverses», et «l'empreinte de la langue du questionnaire» (Malfatto, 2013 : 145-148). À première vue, et en français standard tout au moins, le groupe nominal vache stérile est clair et simple à appréhender : il désigne un bovin femelle adulte incapable de se reproduire.

Pourtant, si compréhensible et univoque que puisse paraître le concept de vache stérile, il s'avère, lorsque l'on se penche sur les atlas linguistiques régionaux et leur contenu, que la traduction dialectale de cet intitulé a posé plusieurs problèmes terminologiques et conceptuels. Les niveaux de connaissance et de technicité effectifs au sein des communautés rurales enquêtées ont, souvent, en dépassant ceux véhiculés par le français standard, rendu obsolète le découpage conceptuel employé par les enquêteurs. Pour preuve, la carte 391 de l'ALG intitulée vache stérile, publiée dans le volume II de l'atlas de Jean Séguy, est considérée, en commentaire, comme sans valeur par l'auteur lui-même : «La carte II, 391 ne vaut rien : elle mêle sous le même titre vache stérile des mots qui correspondent à trois notions différentes dont deux au moins [...] sont en général lexicalement distinctes.» (ALG : 391) Sous l'étiquette vache stérile, la réalité dialectale est donc plus complexe.

En effet, la stérilité d'une vache peut n'être qu'une conséquence ou un corolaire secondaire d'un ensemble plus large touchant l'animal dans son ensemble. Pour certains - dont on ne développera pas les motivations ici - l'impossibilité qu'a une femelle de porter la vie sera une caractéristique importante, grave, voire dramatique et fera l'objet d'une catégorisation et d'une désignation particulière. Mais pour d'autres, aux us différents, la stérilité ne méritera pas tant de considération, et ce pourra être l'une de ses conséquences - l'absence de lactation par exemple - qui représentera

1. Voir Dalbera et al. (1992-) ainsi que Dalbera et al. (2012). 
une tare rédhibitoire. Afin de ne pas complexifier l'étude par la multiplication des concepts corrélés, et sans toutefois se priver d'écarts nécessaires, les données analysées ici sont associées, au sein des atlas, à la notion de vache stérile stricto sensu.

\section{Périphrases descriptives de la stérilité}

Avant d'entamer l'étude des substantifs et adjectifs employés en domaine occitan afin de désigner la vache stérile, nous remarquerons que plusieurs locuteurs emploient, à des localités diverses, des périphrases descriptives de ce caractère. Ainsi, la séquence [v'ow pl'y reten'i], recueillie à Varages (ALP, P. 138), décrit une femelle qui ne veut plus retenir, où le verbe retenir renvoie au fait de rester prégnante, de continuer à tenir sa future progéniture. Il est intéressant de noter ici l'emploi du verbe voler vouloir qui conscientise la femelle dans le processus de gestation; jugée coupable de ne pas porter délibérément, elle aura tôt fait d'être écartée du troupeau. Par ailleurs, le syntagme [ret' en $^{\mathrm{p}} \mathrm{pl}$ 'y], littéralement «elle ne retient plus », est employé à Comps (ALP, P. 122) pour évoquer une bête ne parvenant plus à compléter ses gestations, et que l'on considère comme devenue stérile. Le verbe retenir est également présent à Raphèle-lès-Arles (ALP, P. 139) et Isserpent (ALLy, P. 13) où la bête stérile est décrite par une tournure signifiant «elle ne retient pas», sans évoquer une quelconque volonté ou une idée d'évolution de son état.

Deux autres périphrases désignent quant à elles l'incapacité qu'a la femelle de faire un veau. À Réallon (ALP, P. 36) la vache stérile est celle [ke $\mathrm{f}^{\prime}$ aj p'a lu v'cw] qui ne fait pas le veau, tandis qu'à Signes (ALP, P. 160) cette bête [f'a dz'e de ved'ew] ne fait pas de veau. Enfin, à Alloué (ALO, P. 85), la vache stérile ne [pœ pa rãpl'ir], littéralement ne peut pas remplir, description employée pour illustrer le fait qu'elle ne parvient pas à être gravide.

L'emploi de ces diverses périphrases, au-delà des informations qu'elles fournissent quant au matériel lexical utilisé, dévoile l'absence dans les systèmes de termes - adjectifs ou substantifs - désignant la femelle stérile. Cette non-lexicalisation doit coïncider avec une faible familiarité de l'élevage bovin, et, plus encore, de ses particularités zootechniques; il serait douteux de penser que la stérilité ait été supprimée chez telle ou telle race bovine au point d'avoir contribué à effacer l'item lexical employé pour la nommer. La localisation géographique de toutes ces données nous conforte d'ailleurs dans l'idée selon laquelle il s'agit avant tout d'une non pertinence catégorielle; la faible présence de l'élevage bovin en une partie de la Provence est pointée du doigt par les auteurs de l'atlas qui précisent 
que : «[...] une vaste région, s’étendant du Vaucluse au sud des AlpesMaritimes et couvrant la majeure partie des Alpes de Haute-Provence, le Var et les B. du Rhône, n'[a] pas d'élevage de vaches, traditionnellement.» (ALP : 671)

\section{Mâle reproducteur, femelle nymphomane et stérilité}

Le corpus étudié montre que la vache stérile est souvent qualifiée de turca - ou sa variante turga (Alibert : 683) - adjectif parfois substantivé possédant les sens de «bréhaigne, stérile, impuissant» et n'entretenant pas, à première vue, de rapport étymologique spécifique avec les bovins. Il s'agit d'un terme générique qualifiant tout ce qui ne produit pas, et on le retrouvera par exemple associé à d'autres animaux domestiques tels la brebis ou la chèvre stériles. Mais, malgré ces attestations associant turca et le signifié générique stérile, il apparaît que, diachroniquement, ce type lexical appartient à la famille étymologique du lat. TAURUs taureau (FEW 13.1 : 132 a) et que les variantes phonétiques qu'il représente - telles [t'yrgo], [t'yrdzo] ou encore [t'yrzo] - proviennent d'une base *TAURICCA (Rohlfs, 1978 [1931] : 143-144, note 2), dérivée de TAURUS.

L'utilisation d'une dénomination du mâle adulte, adaptée au féminin, pour désigner la femelle stérile est un phénomène fortement répandu. Ainsi, un type lexical taura continuant le lat. TAURA vache stérile (DELL : 677), d'ailleurs employé dans le texte latin de Columelle en épigraphe de ce travail, est largement attesté au sein des atlas. De même, et comme le montre la carte $n^{\circ} 1$, un dérivé taurèla désigne la vache stérile en de nombreuses localités ${ }^{2}$. Enfin, appartenant également à cette famille étymologique, seront cités les trois hapax [tøyrəl'øzo], [turj' $\mathrm{yro}$ ] et [tur'ijo] respectivement localisés à Usson-en-Forez (ALLy, P. 58), Montauroux (ALP, P. 54) et La Besse (ALMC, P. 53). La carte $\mathrm{n}^{\circ} 1$ fournit la localisation de l'ensemble de ces dénominations appartenant à la famille de TAURUs et permet notamment de remarquer la faible répartition des continuateurs du lat. TAURA vache stérile en comparaison des dérivés qui en sont issus.

En tant que processus de création lexicale prolifique, le rapprochement de la femelle stérile et du mâle reproducteur transcende la variation lexicale. Ainsi, plusieurs attestations n'entretiennent pas de rapport morphologique

2. Il convient de préciser que taurèl, bien que morphologiquement diminutif car continuant le lat. TAURELLU jeune taureau, désigne, sur une grande partie du domaine occitan - à l'exclusion de la Gascogne et d'une partie du Massif central - l'animal adulte. 
avec un nom du taureau appartenant à la famille du lat. TAURUS, mais avec une autre appellation dialectale de l'animal : le type $\mathrm{brau}^{3}$. C'est notamment le cas pour la donnée [e $\beta \mathrm{r}$ 'awdo], littéralement «elle est brauda», qui, attestée à Concots (ALLOc, P. 46.33), décrit un femelle qui serait devenue un brau. De même, l'adjectif [ęmbraw'iðœ], localisé à Ourde (ALG, P. 689SE), dévoile par sa formation - présence du préfixe inchoatif em- et forme de participe passé - un hypothétique verbe *embravir évoquant l'idée d'une femelle qui se serait, là aussi, transformée en mâle.

Cette vision d'une femelle devenue un taureau, est à-même d'expliquer

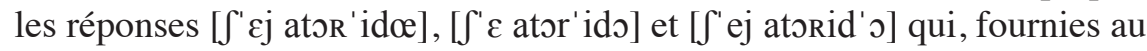
sein de l'ALAL où elles sont glosées elle s'est ataurelée par les auteurs de l'atlas, présentent un participe passé d'un hypothétique verbe pronominal * s'ataurir au sens de se transformer en taureau. De même, la réponse [al storn ã toRẽn] fournie à Nouzerolles (ALAL, P. 35) décrit littéralement une femelle qui se tourne en taureau. La carte $\mathrm{n}^{\circ} 2$ permet la localisation de ces informations supplémentaires. On remarquera l'écart géographique formé par les données morphologiquement associées à brau taureau; leurs formations, leur faible fréquence, cette répartition et le fait que la dérivation prenant pour base le nom du mâle reproducteur pour aboutir à un nom de la femelle stérile soit lexicogénique, plaident en faveur de créations lexicales spontanées.

Mais ces données et leur analyse linguistique n'expliquent pas les raisons de tels choix lors de la création lexicale. Pourquoi, dès le latin semblet-il, la vache stérile est-elle cognitivement rapprochée du taureau, animal reproducteur par excellence, fertile s'il en est? Peut-être Festus (Savagner, 1846, XVIII : 636) nous donne-t-il une piste à explorer lorsqu'il analyse le lien entre taura et taurus du fait que ces femelles «ne vêlent pas plus que les taureaux». Si l'on accordait crédit à cette justification qui ne pourrait être que parétymologie, on considèrerait alors que le mâle ne pouvant, par définition, pas être gravide, les femelles qui n'ont pas, elles non plus, la capacité de l'être en sont rapprochées.

Sachant que la stérilité telle que nous l'entendons est une conception moderne qui ne trouve pas de parallèle dans la vision antique du processus de reproduction, il est en effet permis de prendre en considération la vision de Festus. L'adjectif lat. sTERILIs possède d'ailleurs le sens, avant tout autre, de «animal qui ne produit pas de petit» (DELL : 647), définition qui ne

3. C'est un autre sujet que l'étymologie de brau taureau. Traditionnellement, il est affirmé que ce terme continue le lat. BARBĂRUs peuple de langue étrangère, barbare (DELL : 66). 
précise en rien si la bête pourrait se reproduire ou si elle en serait incapable. Ce qui importait pour les romains, c'est que l'animal ne puisse pas, à un instant donné ou de manière générale, porter la vie, mettre bas, faire partie des bêtes productives, rentables par leur propension à se perpétuer. Nous ne serons donc pas étonnés d'apprendre que des mâles entiers, pourtant aptes à la reproduction, ont pu être qualifiés de sTERILIs du fait de leur incapacité à entrer en gestation et à enfanter ${ }^{4}$. D'ailleurs, pour juger de la fertilité ou de la stérilité d'un individu, le locuteur n'a pas d'autre choix que de se fier à des preuves physiques palpables ou décelables à l'aide des avancées techniques ou médicales de son époque; et, dans le cas présent, la seule preuve tangible de la stérilité d'un animal reste son incapacité à entrer en gestation. Il est donc logique que mâles reproducteurs et femelles stériles aient été considérés d'une manière similaire puisque ne pouvant, ni l'un ni l'autre, porter la vie et mettre bas. De là, le lien qui unit femelle stérile et mâle reproducteur devient intelligible. Reste que l'absence de dénomination dérivée du nom du bøuf - également incapable de mettre bas - peut paraitre suspecte.

Mais il est une particularité de notre corpus de données occitanes qui nous incite à une analyse plus poussée sur la question. En effet, la réalité dialectale telle que fournie par les informateurs des atlas atteste de la présence d'une nuance opposant les concepts de vache devenue stérile et de vache stérile par conformation - ou de naissance - et montre, dans le même temps, une différenciation lexicale dans la manière de les désigner. Ainsi, et c'est de ce point que découle l'analyse qui suit, lorsque les deux types de vaches stériles sont différenciés, le concept associé à une femelle n'ayant jamais pu avoir de progéniture n'est pour ainsi dire quasiment jamais désigné à l'aide de termes morphologiquement associés aux noms du mâle reproducteur, tandis qu'inversement, seules les vaches devenues stériles présentent une dénomination en lien avec le nom du reproducteur. Pour reprendre la vision évoquée plus haut, les vaches perdant leur capacité à se perpétuer deviennent des taureaux, tandis que celles stériles de naissance sont qualifiées à l'aide de termes génériques tels estèrla, mula ( $<$ lat. MULA mule, voir infra) ou bien turga, ce dernier ayant perdu son caractère motivé pour n'être plus qu'un générique signifiant stérile.

D'après les précisions issues des atlas, la stérilité acquise peut avoir plusieurs causes telles la vieillesse, le refus de l'accouplement ou encore

4. Ernout et Meillet (DELL : 647) indiquent à ce propos que «les coqs sont qualifiés de steriless par Varron» et, plus loin, que «dans les emplois anciens, la valeur de qui ne produit pas de petit est encore présente». 
une soi-disant maladie nommée la nymphomanie dont les causes, inconnues du simple paysan, produisent des effets redoutés. Quoiqu'en ces termes elle soit un mythe pour les vétérinaires modernes, la nymphomanie bovine entraine chez la femelle un retour continuel aux chaleurs - dû à une déformation des organes reproducteurs - qui l'empêche de conserver un fœetus viable et la contraint à revenir constamment en état d'œstrus (Berger, $2008: 20-21)^{5}$. La femelle en question sera donc constamment en rut et présentera continuellement tous les symptômes et comportements caractéristiques de cette période généralement très espacée chez un animal pour ainsi dire normal. Du Roizel-Marlier (2004 : 14) décrit cet individu de la façon suivante : «maigre, très active, agressive, meuglant, chevauchant les autres [et qui], toujours en état d'œstrus, [...] tourmente ses congénères, les chevauche et jette le désordre dans le troupeau».

Une vision semblable est ainsi attestée à Uzer (ALG, P. 696E) où l'informateur précise, concernant ce concept de vache nymphomane, que ses nerfs cassent et elle devient comme un taureau. De là, il est envisageable de penser que les dénominations ou adjectifs désignant la stérilité et entretenant un rapport étymologique ou morphologique avec un nom du mâle reproducteur aient pour origine l'existence, au sein des troupeaux de bovins, de femelles présentant un comportement jugé masculin et considéré comme déviant. Les termes en question auraient donc bien pour motif un rapprochement d'avec l'animal reproducteur, mais non car il porte aussi peu qu'une femelle stérile, mais du fait que tous deux aient un comportement identique et nécessitent d'être écartés du troupeau. La question peut également se poser pour le terme lat. TAURA, celui-ci désignait-il réellement (toute) vache stérile ou le peuple de paysans que forment les Romains avait-il déjà établi une distinction entre stérilité acquise et stérilité innée ? Le terme permet de supposer qu'il s'agissait déjà de désigner la vache au comportement de taureau, celle qui, en plus de ne pas pouvoir porter, se conduisait comme un mâle en rut. D'ailleurs, un rapide détour sémasiologique auprès des continuateurs du lat. TAURA nous informe que leur extrême majorité désigne non la vache stérile mais la vache nymphomane ou la vache devenue stérile.

5. Chose que ne fait pas une femelle née stérile puisqu'elle ne peut biologiquement pas entrer en état d'œstrus pour y revenir continuellement. Ceci explique que les descriptions de ces vaches nymphomanes faites par les paysans et utilisées dans divers atlas attestent qu'elles ont déjà porté mais n'y arrivent plus. 


\section{Mulet, vide et stérilité}

En dehors de ces vaches stériles car devenues nymphomanes et des dénominations qui trouvent leur source dans le rapprochement entre ces femelles et le mâle reproducteur, le corpus étudié présente plusieurs données associées à la famille étymologique du lat. vaCARE être vide (DELL : 710). Du lat. vacĩva - féminin de vacĩvus lui-même doublé de vacuUs - proviennent les formes de type vaciva employées en domaine occitan pour désigner la vache stérile. L'image d'un animal vide motive ici l'utilisation de ces termes pour désigner la vache stérile, et ce même si, intrinsèquement, le fait d'être vide et donc non gravide n'implique pas nécessairement la stérilité telle que nous l'entendons ou la définissons. On retrouve là le cheminement de pensée évoqué précédemment, et selon lequel l'absence de gestation équivaut à la stérilité.

D'ailleurs, lors de l'analyse sémasiologique, l'on voit que vaciva peut également désigner la génisse, une bête trop jeune pour se reproduire et non incapable de le faire ${ }^{6}$. Les continuateurs de vacĩva employables pour désigner toute bête ne produisant pas se sont spécialisés, dans le premier cas, pour nommer la femelle vide de manière déviante, et, dans le second, celle qui reste vide de manière régulière ${ }^{7}$. Si chez les continuateurs de l'adjectif lat. sTERILIs l'ancienne vision productiviste s'est perdue, elle perdure chez ceux de vacĩvus qui peuvent désigner la vache stérile ou la génisse future mère. Seule la possibilité de multiplication est prise en compte dans cette conception des bovins domestiques.

Un autre procédé utilisé pour désigner ou qualifier la vache stérile sera l'emploi de termes issus du lat. MŨLA mule. La stérilité est ici évoquée à l'aide de la dénomination d'une bête domestique stérile par définition et devenant ainsi le parangon de cette caractéristique : qui pense à la mule pense à sa stérilité. Le nom de l'animal peut donc être utilisé pour évoquer la femelle bovine incapable de se reproduire ainsi que, plus généralement, toute bête ou chose improductive. Et si la connotation négative associée à la stérilité n'est pas assez claire dans ce genre de dénomination, le lexique

6. Biologiquement parlant, la génisse n'est pas trop jeune pour se reproduire, mais il était de coutume, si l'on en croit les agronomes latins, d'attendre que la femelle ait atteint un certain âge avant d'entamer les premières reproductions. Ainsi, le fruit récolté serait de meilleure qualité que si la femelle s'était accouplée dès ses premières chaleurs. On voit ici que le concept de génisse, purement zootechnique, n'a aucun lien avec une quelconque étape dans l'évolution physiologique de l'animal.

7. Trop jeune ou considérée comme telle et donc écartée du processus de reproduction. 
peut, en lui associant un suffixe péjoratif, renforcer ce caractère; c'est ainsi que l'on rencontre, à Ferrières/Arbéost (ALG, P. 693NE), la forme [myl'atro] vache stérile, dérivée à l'aide du suffixe -astre (Alibert, 1993 : 31-32). La réflexion est la même en ce qui concerne la forme [basi $\beta$ 'arðo], localisée à Lescure (ALG, P. 790NE), qui atteste de l'emploi d'un suffixe péjoratif associé à la base vaciva afin de qualifier cette femelle considérée comme ayant peu de valeur. La carte $\mathrm{n}^{\circ} 3$ permet de voir la fréquence et la répartition géographique de tous les termes mentionnés ici. Concernant les formes rattachées au lat. sTERILIs, il est fort probable qu'elles ne soient pas autochtones mais induites par la langue de l'enquête, et qu'elles ne soient qu'une version patoisante du fr. stérile employé dans les questionnaires.

\section{Rousseur, blancheur et stérilité}

Une autre manière d'évoquer le caractère stérile de l'animal sera l'emploi de l'adjectif blanca. Attestée de manière hapaxique à Eyguières (ALP, P. 130), la forme [bl'ãko], à rapprocher de la racine germanique *blank blanc (FEW, 15.1 : 138b) d'où sont notamment issues les formes rencontrées en français dans tirer à blanc, faire chou blanc ou encore passer une nuit blanche, pointe du doigt le caractère inutile, vide, improductif de l'animal stérile. Ici, la vache stérile est blanca car elle ne produit rien, et, derrière cette donnée, on retrouve l'idée selon laquelle «le blanc est associé à l'absence, au manque» (Pastoureau \& Simonnet, 2005 : 49). Ici, la progéniture manque, et comme la finalité d'une reproductrice est de se perpétuer, si elle n'y parvient pas, elle est considérée comme ratée, comme blanche.

La forme [rim'al], attestée en trois localités situées au nord-ouest du domaine étudié, peut être rapprochée du terme masculin rimèl attesté dans le $\mathrm{TdF}$ au sens de «homme bon à rien» et que Mistral $(2,793 \mathrm{~b})$ rattache au verbe rima possédant plusieurs sens divers dont ceux de roussir; brûler; rider; gercer. Il est envisageable que la vache stérile, rapprochée de cet ensemble sémantique pour les raisons que l'on connait désormais, ait été nommée ainsi car considérée comme roussie, desséchée et donc improductive. Nous rappellerons ici, et de manière succincte, que la vache tarie, celle-là même qui ne donne plus de lait, peut parfois voir ses dénominations associées à celles de la vache stérile, l'incapacité à se reproduire allant de paire avec l'absence de lactation. Animaux bons à rien, la vache stérile et la vache tarie peuvent être désignées à l'aide de termes en lien avec un ensemble d'autres items lexicaux associés à la brulure, la sécheresse ou à leurs conséquences. 


\section{Hermaphrodisme et stérilité}

Nous avons mentionné précédemment la distinction faite par certains locuteurs coutumiers de l'élevage bovin entre les concepts de vache devenue stérile et de vache stérile par conformation. La première possède de nombreuses dénominations issues du nom du taureau, tandis que la seconde est généralement qualifiée à l'aide d'un terme au sens générique de stérile. Mais l'on rencontre également d'autres appellations de la vache stérile par conformation, et notamment les formes [bew v'atso] et [bjow v'atso] attestées dans le Massif central, à Recharenge (ALMC 4) et Saint-Martinde-Valamas (ALMC 7). Ces dénominations de l'animal né stérile montrent un lien morphologique avec le nom de l'animal châtré puisque, dans chacune d'entre elles, la première partie renvoie à un continuateur autochtone du lat. BOVEm boeuf, tandis que la seconde réfère au nom de la vache, issu du lat. vACCA.

La construction de ces termes, présentant la collocation d'un nom de mâle et d'un nom de femelle, n'est pas sans rappeler la matrice lexicogénique employée pour forger des noms d'animaux hermaphrodites. Au-delà de la morphologie du terme fr. hermaphrodite lui-même, notons la présence du type galgalina qui, largement attestée au sein de notre domaine, est composé des noms du coq, gal, et de la poule, galina, et peut désigner, selon les localités, la poule qui chante le $\operatorname{coq}^{8}$, le chevreau hermaphrodite, ou encore notre vache stérile par conformation. De même, plusieurs sources dialectales présentent le type lexical cabriboc composé du nom de la chèvre et de celui du bouc et désignant ici le chevreau hermaphrodite, là la vache stérile par conformation ${ }^{9}$.

Sachant que, dans l'immense majorité des cas, des bêtes présentant des anomalies des organes sexuels sont incapables de se reproduire, alors le lien entre hermaphrodisme et stérilité sera considéré comme véridique et rien n'empêchera plus la vache stérile par conformation d'être nommée à l'aide d'un terme issu du nom d'un animal hermaphrodite ou forgé en suivant la même matrice. Ici encore, il convient de s'affranchir des considérations modernes quant au genre, à l'identité sexuelle des individus ou aux capacités reproductives d'espèces hermaphrodites pour saisir la vision du monde à l'œuvre. Seule la conformité compte en ce qui concerne les bêtes d'élevage, et un individu présentant des organes génitaux non conformes

\footnotetext{
8. Autrement dit un animal hermaphrodite ayant «l'aspect d'une poule, mais les manières d'un coq» (Gardette, 1958 : 172).

9. Le timbre de la seconde voyelle de cabriboc peut s'expliquer par l'opacification du composé cabra e boc > cabreboc > cabriboc.
} 
ou des attributs à la fois masculins et féminins sera aisément taxé d'hermaphrodisme et de stérilité. Le lien entre hermaphrodisme et stérilité, effectif au sein du lexique, l'est sans conteste également dans la vision du monde partagée par les locuteurs; le FEW (22.1 : 247b) atteste d'ailleurs pour le terme rimal évoqué plus haut des sémantismes renvoyant à des animaux hermaphrodites qui, selon nous et suite à la découverte de particularités physiques de leurs organes reproducteurs, ont été considérés comme défectueux, peut-être roussis ou desséchés, et stériles.

$\mathrm{Au}$ sein de l'ALP, nous rencontrons, à Belvédère (ALP, P. 85), la vache stérile par conformation associée à la séquence [es ag'y galgal'i] littéralement $i l$ a eu galgalin. Le terme galgalin est ici un adjectif masculin permettant de décrire un animal qui serait né hermaphrodite et donc stérile. Le fait que le masculin désigne la vache stérile s'explique si l'on considère que le syntagme se réfère à un animal de sexe indéterminé. La présence du verbe aver «avoir» laisse supposer que l'hermaphrodisme est considéré comme une maladie ou une tout autre affection pouvant s'attraper. Lorsque l'éleveur examine la bête après la naissance, il s'aperçoit que le veau est de sexe indéterminé, il en conclut que l'animal a eu galgalin, autrement dit qu'il a eu l'hermaphrodisme; dans l'esprit de l'homme, cette caractéristique associe dès lors l'animal à la stérilité.

À la localité Le Monastier (ALMC, P. 23), c'est la forme [dzaj'ino], continuant semble-t-il le lat. GALLINNA poule (DELL : 266), qui est employée comme substantif pour nommer la vache stérile par conformation. Plutôt que de tenter une explication hasardeuse faisant du nom de la poule celui de la vache, nous pensons que cette attestation présente la déformation du type galgalina suite à sa démotivation, son opacification et son utilisation conventionnelle ${ }^{10}$. La trace d'un continuateur du lat. GALLINNA poule plaide d'ailleurs en faveur soit d'un emprunt soit d'une certaine ancienneté du terme puisque, en ce système, la poule n'est pas nommée galina mais pola $(<\text { PǓLLA })^{11}$.

10. Sur les étapes que suit le signe linguistique depuis sa création lexicale, nécessairement motivée pour être accepté par la communauté, en passant par son utilisation conventionnelle d'où peut découler son caractère opaque, voir notamment Guiraud (1986) et Dalbera (2006).

11. En cette zone, les continuateurs du lat. GALLĩna poule ont été évincés par pola (BrunTrigaud, Le Berre \& Le Dû, 2005 : 297, c. 442). Il sera intéressant de comparer la situation onomasiologique du concept de poule avec toutes les données disponibles concernant les mammifères domestiques considérés comme hermaphrodites. Nous verrions certainement, si tant est que les données soient suffisamment nombreuses, les empreintes laissées par le lat. GALLĩna poule dans le lexique. 


\section{Tératogenèse et stérilité}

Concernant les bovins, il apparaît que, dans le cas des gestations doubles à jumeaux de sexes différents, «le veau femelle est infertile en raison du sous-développement de ses organes sexuels» puisque «sous l'effet des hormones protéines mâles, les cellules provenant du frère jumeau ont inhibé la formation des organes sexuels chez sa sœur jumelle» (Berger, 2009 : 26-27); de fait, la velle jumelle d'un veau présentera des anomalies et aura tôt fait d'être considérée comme stérile par conformation et d'être nommée à l'aide d'une appellation concaténant le nom d'un mâle et celui d'une femelle.

Un informateur, à La Chapelle-en-Vercors (ALP, P. 11), à d'ailleurs fourni la précision suivante «quand il y a deux veaux jumeaux, l'un d'eux est [зym'are]», terme traduit par hermaphrodite ${ }^{12}$. Le lien entre grossesse gémellaire et hermaphrodisme est donc connu du simple paysan.

Ces informations, quoi qu'apparemment anecdotiques, nous entrainent sur les traces de Pierre Gardette, responsable de l'ALLy, qui, en 1958, dans le volume en l'honneur de Gerhard Rohlfs, écrivait un article relatif au fr. jumart, désignant cet animal fabuleux issu du croisement des espèces bovine et chevaline (TLFi), dont il retraçait l'histoire. En conclusion de son brillant hommage, Gardette écrivait ceci :

Et notre histoire est finie! Sans doute des documents nouveaux que des enquêtes nouvelles ne manqueront pas d'apporter, permettront de préciser certaines étapes de cette histoire. Ils fourniront peut-être le jumar paysan qui nous manque; ils poseront certainement d'autres questions. Ce qui rend passionnantes les recherches sur les mots du passé, c'est qu'ils n'ont jamais fini de nous proposer des énigmes.

L'élaboration ultérieure de l'ALP, document nouveau issu d'enquêtes nouvelles, exaucera les vœux de Pierre Gardette en nous permettant, au détour d'une carte intitulée la vache stérile, de mettre la main sur la forme [3ym'are] faisant défaut à la phylogenèse du terme français jumart.

Se basant sur quelques attestations donnant foi à l'existence d'un hybride monstrueux issu là de l'accouplement d'une vache et d'un âne, ici de celui d'un taureau et d'une ânesse, Gardette (1958 : 176) propose que les termes dialectaux qu'il étudie et regroupe - et qui désignent divers êtres hybrides, hermaphrodites, stériles ou difformes - soient les descendants d'un terme massaliote $\chi \dot{\mu} \mu \alpha \iota \rho \alpha$ désignant la chimère, bête fabuleuse

12. La présence de [3ym'arc] est également attestée à Jaillans (ALP 7) où le terme est associé au concept de vache hermaphrodite. 
à corps de chèvre, à buste de lion, à queue de serpent. Évoquant en note les difficultés phonétiques que posent cette étymologie, Gardette estime que le terme $\chi i \mu \alpha \imath \rho \alpha$ a servi à désigner ce référent problématique «en passant de la bouche des marchands massaliotes beaux parleurs dans celle des paysans de la côte, amusés, sceptiques, et aimant déjà sans doute les bonnes plaisanteries».

Désireux de transposer les légendes helléniques à leurs fermes sans prétentions, les autochtones auraient intégré à leur lexique le terme nouvellement venu afin de l'associer à une bête dépréciée et difforme commune dans le paysage agricole. À partir du signifié produit de l'union du taureau et de la jument, les termes issus de $\chi \dot{i} \mu \alpha \imath \rho \alpha$ auraient pris ceux de chevreau hermaphrodite, femelle stérile, enfant insupportable ou encore femme hommasse.

À la lumière des faits évoqués précédemment, ces explications nous laissent dubitatifs. Tout d'abord, car elles se basent sur une prétendue croyance en l'existence, au sein de la ferme et donc dans un cadre quotidien bien connu, de monstres fabuleux issus de croisements improbables; ensuite, car elles évoquent des représentations stéréotypiques, par définition bien peu raisonnables, peignant d'un côté des massaliotes éloquents et cultivés, et, de l'autre, des méridionaux allègres quoiqu'un peu rustres et avides de culture exogène; encore, car il ne nous semble pas, de notre modeste expérience du fonctionnement du lexique relatif aux animaux domestiques d'élevage, qu'une quelconque population de paysans ou d'éleveurs ait eu besoin, envie ou même intérêt à associer une bête défectueuse car stérile et difforme à un animal de légende ou de mythe, ni même à forger des fables autour d'elle; enfin, car elles laissent de côté les savoirs zootechniques de toute une population pour leur suppléer des considérations esthétiques ou facétieuses. Bien que plaisante et attestant de la survivance d'un mot grec en terre française (Gardette, 1958 : 165), l'hypothèse ne peut nous satisfaire.

De notre point de vue, les populations pratiquant l'élevage ont, de tout temps et hors de toute considération fabuleuse, eu affaire à des animaux difformes ou malformés, défectueux ou déviants ${ }^{13}$. Ces animaux, nous l'avons dit, auront tôt fait d'être mis à l'écart, au rebus, catégorisés d'une manière différente de leurs congénères et, de ce fait, désignés par des termes spécifiques permettant de ne pas les associer au reste du troupeau.

13. Parmi les divers bovins difformes à la naissance, il est commun de rencontrer et de distinguer monstres simples et monstres doubles, nommés bijumeaux. L'institut de l'élevage, dans un ouvrage relatif aux maladies bovines, avance le chiffre de $3 \%$ de naissances tératologiques chez cette espèce. 
Parmi les bovins domestiques, la science moderne a prouvé que les grossesses gémellaires étaient productrices d'individus non conformes, aux organes génitaux atrophiés et, parfois, aux caractéristiques tout simplement déviantes, pour ne pas dire monstrueuses. Là où nous nous écartons de l'hypothèse de Gardette, c'est que nous croyons que les populations en question ont eu connaissance, à leur niveau et incluant donc des catégorisations différentes et des confusions sans doute incompatibles avec les observations modernes, du lien entre gémellité bovine et tératogenèse. C'est pourquoi, plus que de penser que le sens premier de jimère fut celui de produit de l'union du taureau et de la jument, nous préférons supposer qu'il fut celui de produit défectueux d'une grossesse gémellaire. De là peuvent aisément découler les signifiés des termes actuels associés à la stérilité, l'hermaphrodisme, la difformité ou la monstruosité, sans avoir à passer par le mystère de l'entité chimérique ou l'attirance des monstres exotiques et lointains. Les monstres dont il est question ici sont tout ce qu'il y a de plus terre-à-terre, de plus tangible, et si le XVIII ${ }^{\mathrm{e}}$ siècle a pu croire en l'existence du jumart en tant que créature fabuleuse, cela doit être par raréfaction de ces individus suite aux perfectionnements de la sélection artificielle et des techniques vétérinaires, plus que par désir d'exotisme.

Du point de vue phonétique, rattacher jimère et ses variantes à la famille étymologique du lat. GĔMǏNUS, GĔMĔLLUS ne pose pas plus de problème

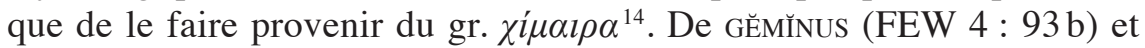
GĚMĚLLUS (FEW $4: 90 \mathrm{~b}$ ) proviennent un ensemble de termes dont les traitements phonétiques du radical concordent avec ceux relevés ici ${ }^{15}$. Et si le cas du radical ne pose pas de problème, celui de la finale pourrait fort bien avoir été influencé par la présence dans le lexique d'un autre terme avec lequel il se serait croisé. À ce propos, et pour boucler cette étude sur les désignations lexicalisées de la vache stérile, nous reprendrons les mots de Pierre Gardette pour avouer qu'en ce qui concerne le jumart l'énigme reste entière.

14. Gardette (1958 : 180, note 1) évoque ceux du genre du terme, qui passerait de féminin à masculin, et celui du $r$ fortement roulé ne pouvant provenir d'un $r$ simple latin. Nous ajouterons le consonantisme initial, qui n'aurait pas dû donner [3], et qui fut justifié en vertu d'un croisement entre les continuateurs du gr. Xí $\mu \alpha \iota \alpha$ de ceux du lat. JÜMENTUM jument (Ronjat, 1932 : $§ 470 \alpha$ ).

15. Sans se douter que des données ultérieures allaient donner un caractère quasiment propitiatoire à ses propos, Gardette s'appuie sur le fr. jumeau pour expliquer le passage de jimère à jumère : «Il faut y voir simplement l'action de la tendance phonétique de l' $i$ à se labialiser en $u$ lorsqu'il est suivi de $m$, surtout lorsqu'il est précédé d'une labiale. Les exemples français jumeau, fumier sont dans toutes les mémoires.» (1958: 177) 


\section{Conclusion}

À l'opposé des bêtes sauvages ou fabuleuses qui peuplent les mythes et les légendes, font les contes et les fables, nourrissent l'imaginaire et inspirent crainte ou vénération, les mammifères d'élevage, individus tangibles, concrets, terre-à-terre, pour ainsi dire réifiés, sont, pour l'homme, l'objet de manipulations réfléchies, de savoirs techniques, de connaissances spécifiques à visée rentabiliste ${ }^{16}$. Au regard de leurs parents sauvages, on pourrait considérer leurs dénominations comme quasiment affranchies, depuis au moins l'avènement des monothéismes, de toutes considérations ésotérique ou mystique ${ }^{17}$.

Pourtant, l'étude du vocabulaire de ce champ lexical n'est pas exempte en enseignements, et la courte étude que nous venons de mener a permis de mettre en lumière, outre des processus linguistiques effectifs lors de la création lexicale, plusieurs rapprochements cognitifs, liens culturels ou ethnologiques, visions du monde, motivations sémantiques qui unissent l'homme et cette bête domestique particulièrement marquée qu'est la vache stérile. Considérée comme un mâle reproducteur pour des raisons éthologiques attestées ou inventées, rapprochée de ce même mâle de par leur incapacité commune à produire, jointe à divers hybrides inféconds ou bêtes hermaphrodites considérées comme anormales ou monstrueuses, la femelle stérile est fortement dépréciée. D'ailleurs, comment pourrait-elle ne pas l'être dans cet environnement où la production est une donnée primordiale, elle qui n'augure que pertes pour son propriétaire? Dans certains cas, nous l'avons vu, la malheureuse sera même soupçonnée de ne pas porter la vie de manière délibérée, et sera jugée responsable de sa condition, comme si elle avait sciemment décidé de se jouer des hommes et de se venger de sa condition. De là à instruire des procès contre sa personne, il n'y a qu'un pas $^{18}$.

16. Nous nous autorisons, pour reprendre l'expression de Danielle Corbin (1989: 35) à propos, notamment, de ce terme rentabiliste, à emprunter les voies de la création lexicale.

17. On ne peut nier la présence de croyances ou de superstitions associées aux mammifères domestiques. Il suffira d'évoquer les tomes IV et V de la Faune populaire de la France d'Eugène Rolland (1881 et 1882) ou les Croyances, mythes et légendes des pays de France de Paul Sébillot (2002) pour se le remémorer. Mais le fait est qu'elles n'ont pour ainsi dire pas de lien avec le mystique ou le religieux - si l'on excepte leur genèse - ou que, lorsque tel est le cas, les animaux domestiques y sont généralement la proie de bêtes sauvages ou l'objet de leurs méfaits.

18. Sur les procès d'animaux, voir notamment Vartier (1970). 
Cognitivement parlant, en ce qui concerne les représentations et les motifs à la création lexicale, on est très loin de la simple description physique ou des ressorts magico-religieux que l'on peut retrouver dans nombre d'analyses de zoonymes d'animaux sauvages. Et si nous avons volontairement limité notre propos à la seule vache stérile, les données dont nous disposons concernant le concept de vache nymphomane sont également édifiantes. Il n'y est plus question de définir la bête comme inutile ou vide, au contraire, le cheminement anthropomorphique y est plus avancé, et la vache nymphomane possède un ensemble de dénominations trouvant leur origine dans celles de diverses femmes de mauvaise vie, prostituées, ribaudes ou hommasses.

En jouant le rôle d'exutoire, la bête domestique déviante canalise le dépit des hommes face à la promesse de pertes sur investissement, de privations, ou de périodes de vaches maigres.

\section{RÉFÉRENCES BIBLIOGRAPHIQUES}

ALAL = PоттE Jean-Claude, 1975-1992, Atlas linguistique et ethnographique de l'Auvergne et du Limousin (3 vol.), Paris, Éditions du CNRS.

ALCe $=$ DuBuIsson Pierrette, 1976-1982, Atlas linguistique et ethnographique du Centre (3 vol.), Paris, Éditions du CNRS.

ALG = SÉGUY Jean, 1954-1974, Atlas linguistique et ethnographique de la Gascogne (6 vol.), Paris, Éditions du CNRS.

ALIBERT Louis, 1993, Dictionnaire occitan-français d'après les parlers languedociens [1966], Toulouse, Institut d'études occitanes.

ALLOc = RAVIER Xavier, 1978-1993, Atlas linguistique et ethnographique du Languedoc occidental (4 vol.), Paris, Éditions du CNRS.

ALLOr = BoIsGONTIER Jacques, 1981-1986, Atlas linguistique et ethnographique du Languedoc oriental (3 vol.), Paris, Éditions du CNRS.

ALLy = GARDETTE Pierre, 1967-1976, Atlas linguistique et ethnographique du Lyonnais (5 vol.), Paris, Éditions du CNRS.

ALMC $=$ NAUTON Pierre, 1955-1961, Atlas linguistique et ethnographique du Massif central (4 vol.), Paris, Éditions du CNRS.

ALO = MASSIGNON Geneviève \& HorIoT Brigitte, 1971-1983, Atlas linguistique et ethnographique de l'Ouest (3 vol.), Paris, Éditions du CNRS.

ALP = BOuvier Jean-Claude \& MARTEL Claude, 1975-1986, Atlas linguistique et ethnographique de la Provence (3 vol.), Paris, Éditions du CNRS.

BERGER Jutta, 2008, «Retours en chaleur toutes les trois semaines», TORO, nº 10/ 08, p. 20-21.

BERGER Jutta, 2009, «Jumeaux à l'étable : une bénédiction?», TORO, nº 04/09, p. 26-27. 
Brun-Trigaud Guylaine, Le Berre Yves \& Le Dû Jean, 2005, Lectures de l'Atlas linguistique de la France de Gilliéron et Edmont, CTHS, Paris.

Corbin Danielle, 1989 «Contraintes et création lexicales en français », L'Information grammaticale, $\mathrm{n}^{\circ} 42$, p. 35-43.

DALBERA Jean-Philippe et al., 1992-, Thesaurus Occitan, 'THESOC', Université Nice-Sophia-Antipolis, CNRS/UMR 7320 BCL, <http://thesaurus.unice.fr>.

DAlbera Jean-Philippe, 2006, Des dialectes au langage. Une archéologie du sens, Paris, Honoré Champion.

Dalbera Jean-Philippe et al., 2012, «La base de données linguistique occitane Thesoc. Trésor patrimonial et instrument de recherche scientifique», Estudis Romànics, no 34, p. 367-387.

DELL = ERnout Alfred \& MeILlet Antoine, 2001, Dictionnaire étymologique de la langue latine. Histoire des mots [1959], Paris, Klincksieck.

Du BoIs Louis-François, 1845, Columelle. De l'économie rurale, Paris, Panckoucke.

Du RoIZEL-Marlier Thibaut, 2004, Effets de l'ovariectomie sur la production laitière des vaches de races Montbéliarde et Prim'Holstein. Étude cas-témoins, thèse de doctorat, École nationale vétérinaire d'Alfort, Maisons-Alfort, France.

FEW = VON WARTBURG Walter, 1922-2002, Französisches Etymologisches Wörterbuch. Eine Darstellung des 31 galloromanischen sprachschatzes, 25 vol., Bonn, Berlin, Bâle, Klopp, Teubner, Zbinden.

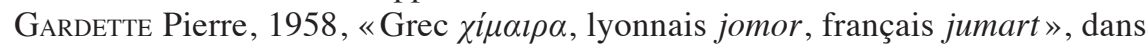
H. Lausberg et H. Weinrich (éds), Romanica. Festschrift für Gerhard Rohlfs, Halle, Niemeyer, p. 166-180.

Guiraud Pierre, 1986, Structures étymologiques du lexique français, Paris, Payot.

Malfatto Albert, 2013, «Perception catégorielle et pertinence référentielle. Le cas des animaux domestiques en domaine occitan », Corpus, $\mathrm{n}^{\circ}$ 12, p. 141-171.

Pastoureau Michel \& Simonnet Dominique, 2005, Le petit livre des couleurs, Paris, Panama.

RoHLFs Gerhard, 1978, «Beiträge zur Kenntnis der Pyrenäenmundarten » [1931], Revue de linguistique romane, $\mathrm{n}^{\circ}$ VII, p. 119-169.

Ronjat Jules, 1932, Grammaire istorique des parlers provençaux modernes, vol.2, Montpellier, Société des langues romanes.

Savagner Auguste, 1846, Sextus Pompeius Festus. De la signification des mots, Paris, Panckoucke.

TDF = Mistral Frédéric, 1979, Lou Tresor dóu Felibrige [1878], Raphèle-lèsArles, Culture provençale et méridionale.

VARTIER Jean, 1970, Les procès d'animaux du Moyen Âge à nos jours, Paris, Hachette. 


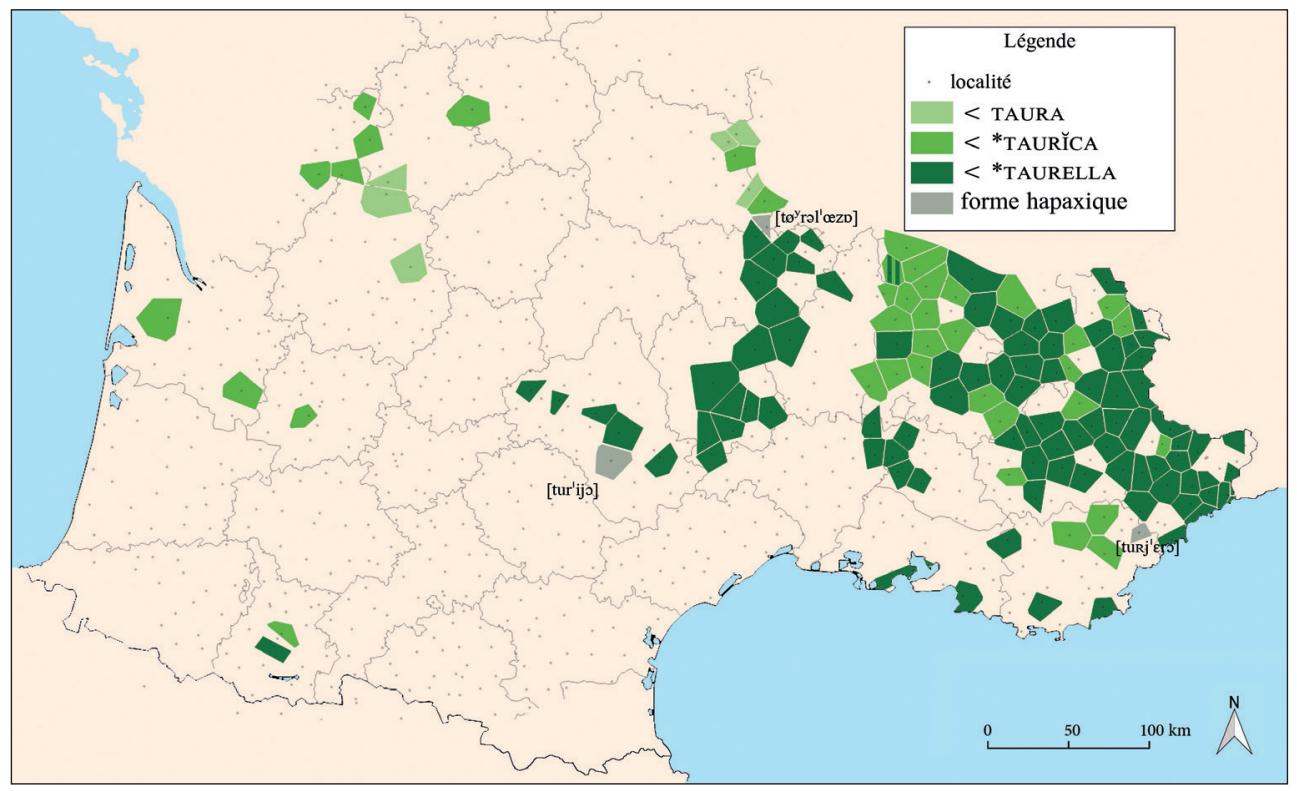

Carte 1. - La «vache stérile», dénominations appartenant à la famille étymologique du lat. TAURUS.

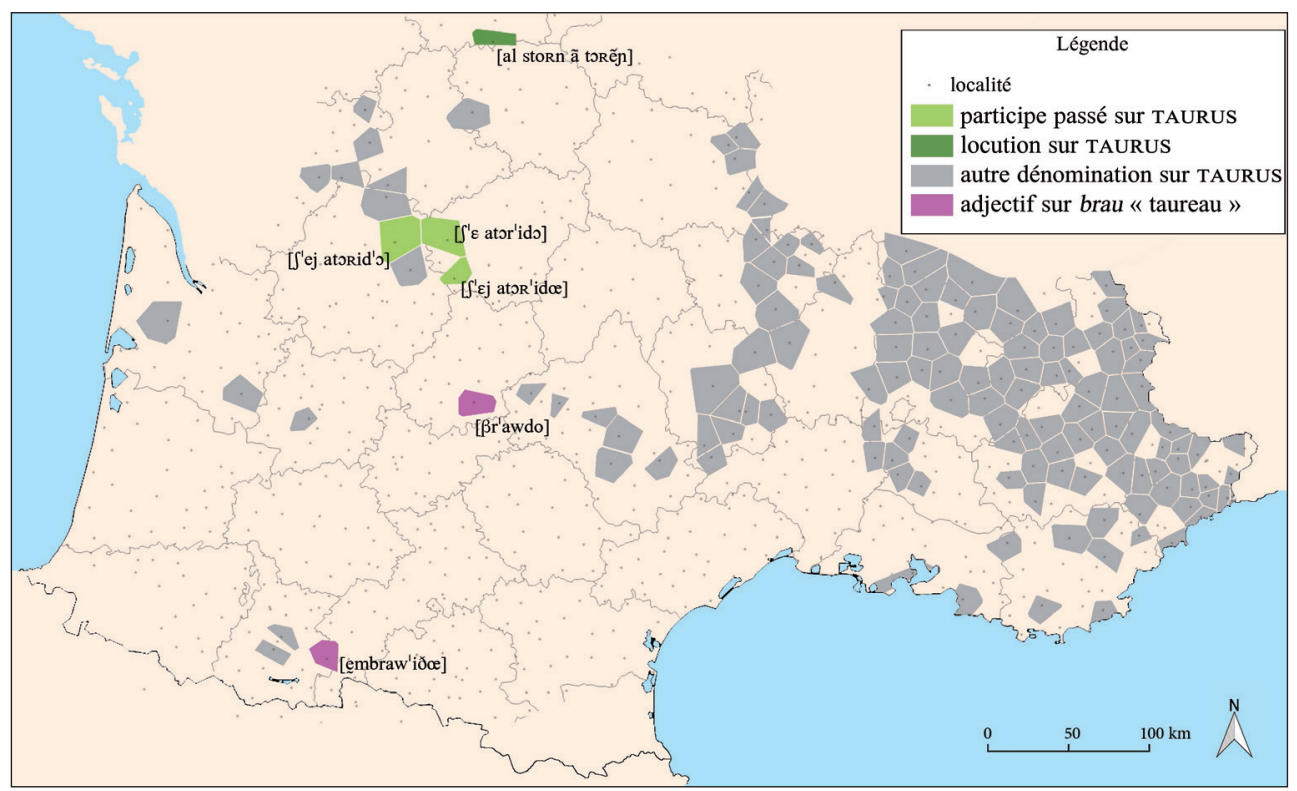

Carte 2. - La «vache stérile», dénominations en rapport avec le nom du mâle reproducteur. 


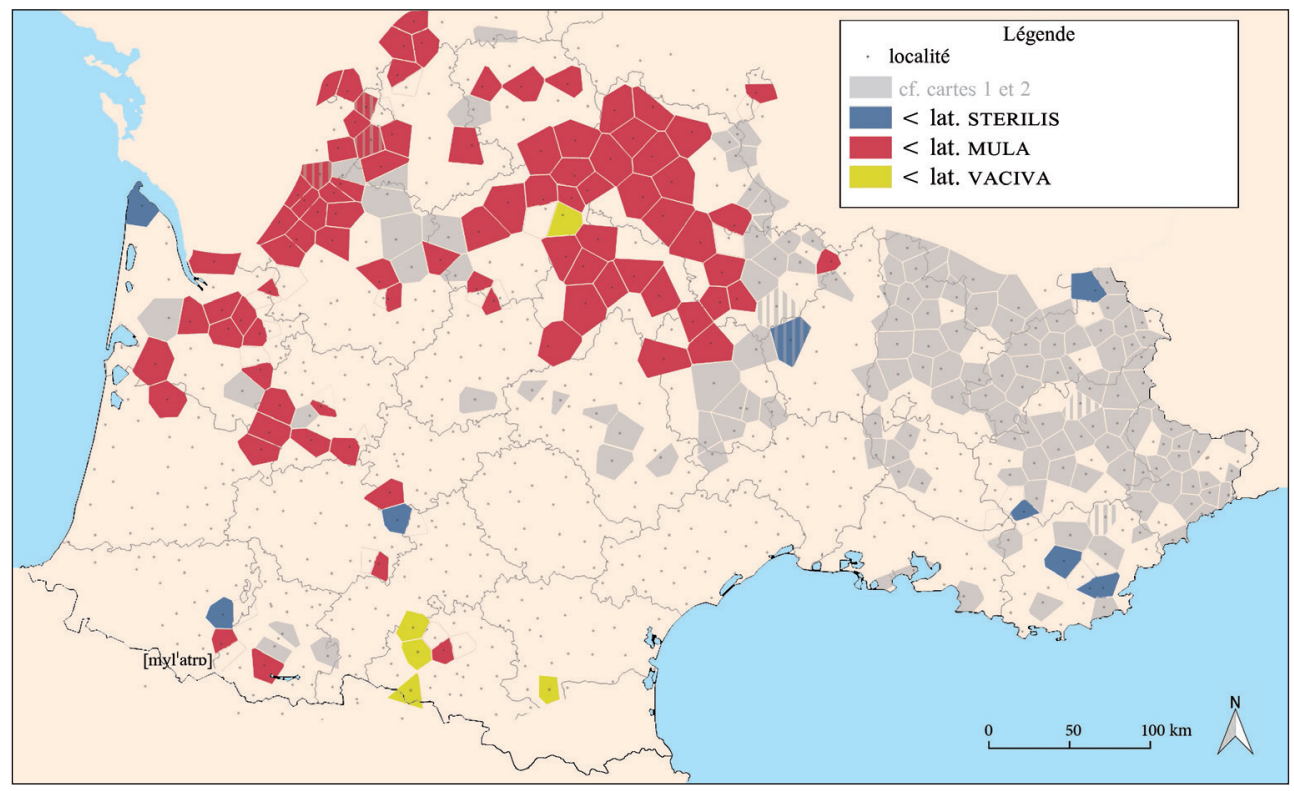

Carte 3. - La «vache stérile»,

dénominations associées au lat. STERILIS, MULLA OU VACIVA.

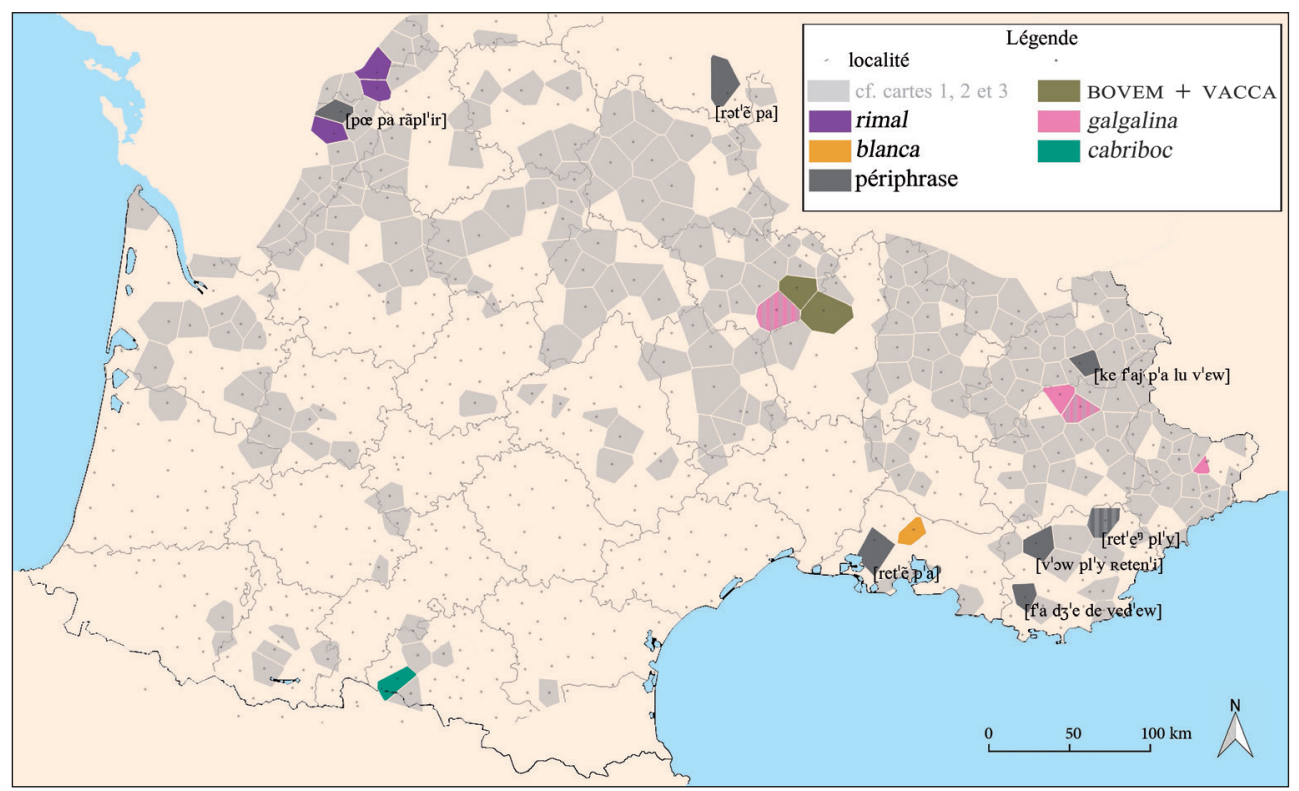

Carte 4. - La «vache stérile», autres dénominations. 